\title{
Development of large aperture elements for active and adaptive optics
}

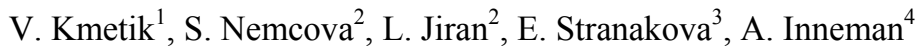 \\ ${ }^{1}$ Institute of Plasma Physics v.v.i., AcSci CR, Za Slovankou 3, 18000 Prague, Czech Rep. \\ ${ }^{2}$ Faculty of Mechanical Engineering, CTU Prague, Czech Rep. \\ ${ }^{3}$ 5M s.r.o., Kunovice, Czech Rep. \\ ${ }^{4}$ Rigaku Innovative Technologies Europe s.r.o, Prague, Czech Rep.
}

\begin{abstract}
Large-aperture elements for laser active and adaptive optics are investigated in collaboration within IOP AcSci CR, FEng CTU and 5M. A bimorph deformable mirror for high-power lasers based on a lightweight structure with a composite core is currently in development. In order to realize a sufficiently large working aperture we are using new technologies for production of core, bimorph actuator and DM reflector. Detailed simulation of components and structure is validated by measurement and testing. A research of DM actuation and response of a complicated mirror structure needed for an accurate control of a deformation is performed. Testing of samples and subscale measurements are currently performed, measurement of a complete structure is in preparation.
\end{abstract}

\section{Laser active and adaptive optics}

Modern pulsed and repetitive lasers are capable to produce coherent light beams with extreme high peakpower as well high average-power. Formation of a laser beam close to diffraction limit and maintaining its high quality is needed for many applications in science, aerospace, defence, energy production and other fields as medicine, industrial technologies and transportation. In practice, the quality of the laser beam is influenced by an operation of laser itself, and by external factors which are often difficult to predict and to control entirely.

A significant research effort is spent today for an accurate adjustment of laser beam and to the correction of perturbations in order to produce and maintain a high beam quality by means of an active and adaptive wavefront control.

In a technique similar to the method used to improve a received image quality in astronomy, a high-power laser active and adaptive optics uses an accurate $2 \mathrm{D}$ phase control for modification of the optical wavefront [1]. High power laser beams may unfortunately cause damage of common optics by high peak and high average power, hence special high damage threshold materials and elements have to be used. Low-power optical phase correctors as wavefront correction devices for large astronomical telescopes are not directly usable for handling of high power laser beams. A sufficiently high resistance to optical damage and reliability requires not only use of special materials for optical surfaces and transparent elements, but it significantly influences internal structures of $\mathrm{AO}$ elements and it affects overall performance. Reinforcing of AO components for a high power use often reduces critical parameters as dynamics range, spatial/temporal response, precision etc. Also phase perturbations to be corrected by the high-power laser AO are usually other than for low power correctors because systems operate at different conditions.

Contemporary low-power AO can use different wavefront control techniques to improve its operational parameters and to reduce a cost of devices. However in case of high-power active and adaptive optics systems currently operated in leading laser laboratories, so far only sufficiently resistant deformable mirrors were able to handle high power of laser beams accurately and reliably enough.

\section{Large aperture laser AO}

Although high-power laser active and adaptive optics is investigated several decades yet, laser AO systems are often custom built devices. Some smaller apertures laser AO systems are today commercially available, however at a rather high cost. Large-aperture high-power AO devices are rare prototypes developed for a few particular applications.

Particular achievement of laser AO was a development of active large-aperture laser-beam correctors for NIF and LMJ fusion lasers [2,3].

This is an Open Access article distributed under the terms of the Creative Commons Attribution License 2.0, which permits unrestricted use, distribution, and reproduction in any medium, provided the original work is properly cited. 


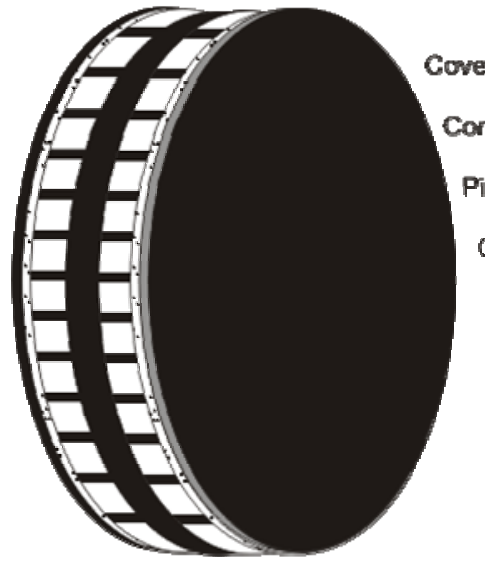

Cover Layer

Control Electrodes 1

Piezo Actuator 1

Composite Core

Piezo Actuator 2

Control Electrodes 2

Thin Mirror substrate

Reflective Low-stress Thin Layer

Fig. 1. The optimized bimorph deformable mirror.

In both cases, an active control for square 300x300 $\mathrm{mm}$ laser beams with intensity over $\mathrm{GW} / \mathrm{cm}^{2}$ was reached by means of massive deformable mirrors mechanically driven by piezo elements and/or electric servomotors. In spite of lower mode corrections only and several second response time, excellent precision, stability and reliability was achieved. Because of stable laboratory environment a large and heavy support necessary for the deformable mirror with a thick glass substrate could be readily used.

In order to reach a higher spatial resolution and a faster response, current AO research is focused to lightweight AO elements with significantly bigger amount of actuators.

A large size adaptive beam corrector with a reduced weight and a high density actuator array for high power cw lasers was developed by AOA Xinetics division of Northrop Grumman Corporation [4].

A promising architecture to reach a sufficiently large aperture with a high spatial frequency actuation ability is based on a bimorph DM structure. Up to date, the largest $25 \mathrm{~cm}$ bimorph deformable mirror designed for $15 \mathrm{~cm}$ beam at the Vulcan petawatt laser was realized in CLF RAL, UK [5].

Larger size high-power bimorph deformable mirror could not be currently build because of lack of necessary components. A big obstacle for further development is also an excessive price of high-power AO. However new solutions for $\mathrm{AO}$ are urgently needed for planned European lasers in HIPER and ELI projects [6].

\section{Advanced scalable bimorph DM}

\subsection{Design, modelling and testing of bimorph DM}

In order to realize large-aperture deformable mirror for high power lasers with sufficiently dense control elements we selected an approach based on economically acceptable bimorph architecture with special composite core and innovative actuation structure permitting larger dynamic range and better precision.

We are developing needed elements and components for a large-size in-house designed DM. It includes optimized flexible composite core, novel bimorph actuator, control array and thin reflector scalable to large apertures. We are investigating also new methods and technologies for connection and integration of elements of DM together into single unit. Fundamental elements of designed large aperture DM are shown in Fig.1.

A particular effort is spent on design, analysis and testing of novel architecture of the bimorph actuator with a low voltage control array.

To reach required performance, we are currently modeling elements of DM and testing suitable materials necessary for optimizing of DM elements, as well as we are investigating new technologies for production of unusual components not available today on the market.

Development process includes measurement of material samples, modeling of parts and components and their strict testing before including into entire element design. Detailed simulation of the elements and assembled units is experimentally verified on subscale models with emphasize to scalability to larger sizes.

Complex numerical model for simulation of DM based on ABAQUS software was assembled and tested on selected samples. Currently we are validating and tuning simulation on samples and fundamental elements. Simulation result of a simple test sample is presented in Fig.2. Validated numerical model is used for calculation of the deformations of assembled elements and scaled models of DM. Fig 3 shows simulation of full-scale

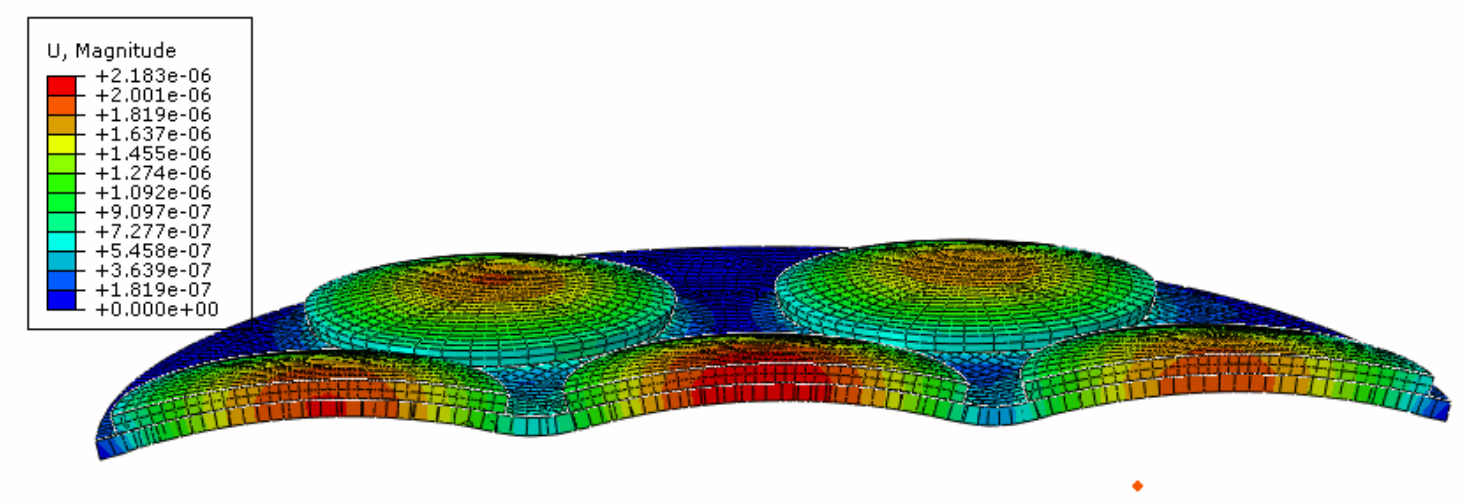

Fig. 2. Small size DM structure simulation. A monomorph test sample driven by uniform 100V voltage on control electrodes 

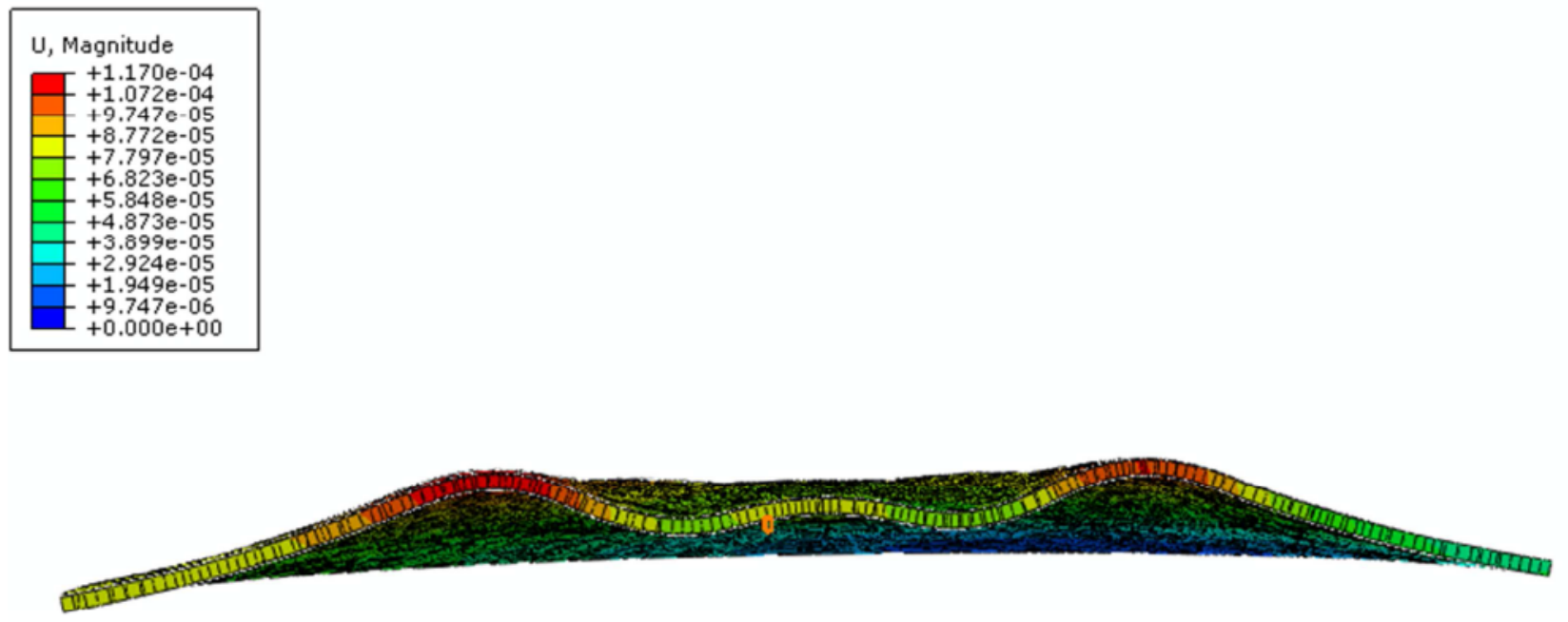

Fig. 3. Simulation of a circular $300 \mathrm{~mm}$ simplified DM model with homogenous actuator layer and 19 control electrodes.

model with 17 electrodes and aperture $30 \mathrm{~cm}$.

Response of components, elements and complex structure is used for an internal structure modification, for experimental models parameter tuning and optimization of the prototype. A final structure of the prototype will be decided with help of both simulation end elements measurement results.

\subsection{Scaling of bimorph DM to larger sizes}

Principal obstacle for realization of large size bimorph deformable mirror is difficulty to produce a homogenous and isotropic layer of an actuator material on a core of the DM without unwanted deformation of entire structure.

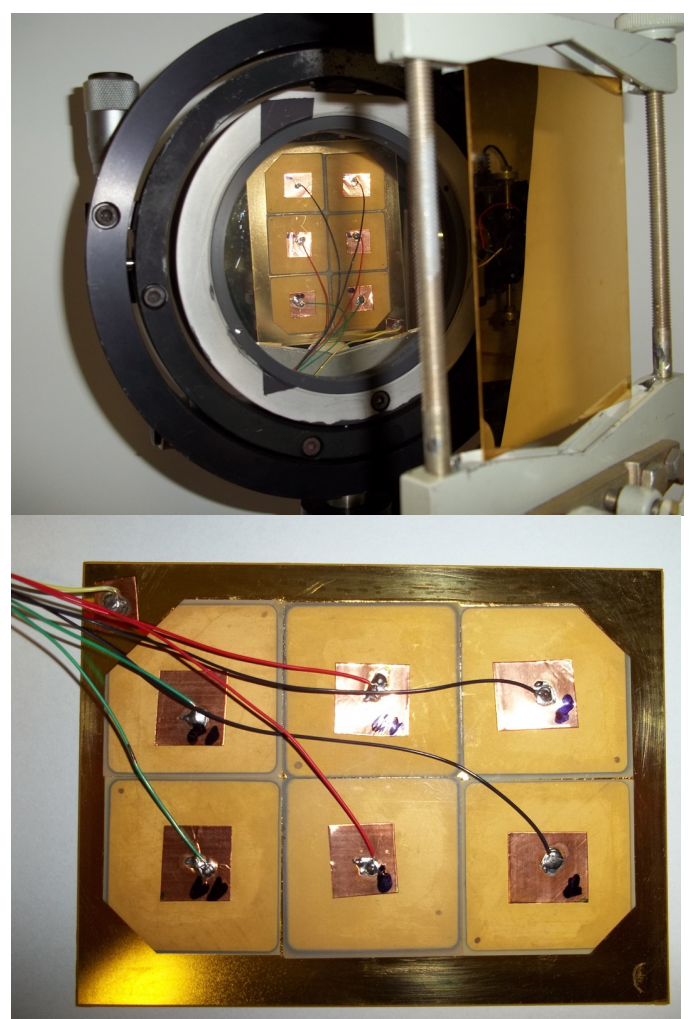

Fig. 4. An early sample of DM used form testing a scalable segmented actuator
Scaling to larger sizes can enable significantly wider use of active and adaptive optics by offering an inexpensive and easy available DM structures.

In this contribution we report on research of bimorph DM using small size piezo-ceramics elements to create a large size a scalable actuator. This technique was selected because it can enable scaling to larger sizes at relatively low cost.

The early sample of bimorph DM used to test segmented actuator was made of 6 individual pieces piezo-elements bonded to $0.6 \mathrm{~mm}$ substrate. A sample used to test the segmented actuator is shown in Fig.4.

While the first test of a sample structure by focusing laser beam showed an encouraging response to control voltage, a detailed quantitative examination of structure

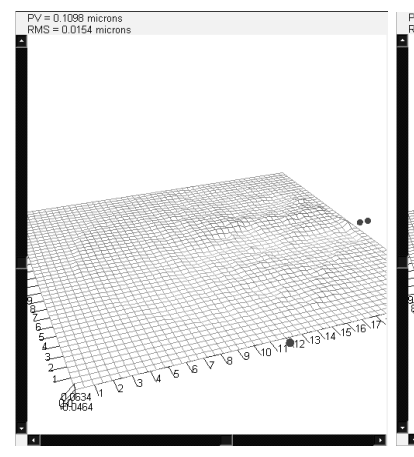

a

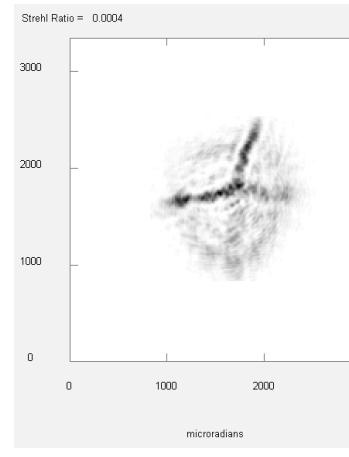

c
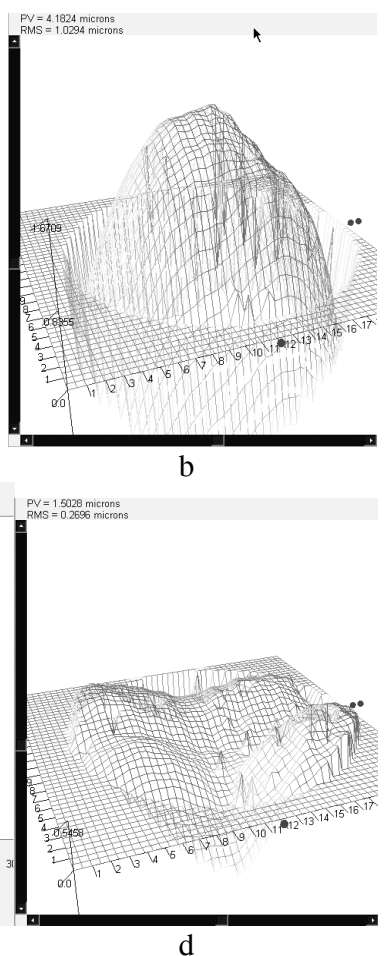

d
Fig.5. Measured wavefront and deviation from a correct surface. a) wavefront at $0 \mathrm{~V}, \mathrm{~b}$ ) wavefront at $75 \mathrm{~V}, \mathrm{c}$ ) calculated far field at $75 \mathrm{~V}$, d) focus removed at $75 \mathrm{~V}$. 
Influence of discontinuities in the actuator layer

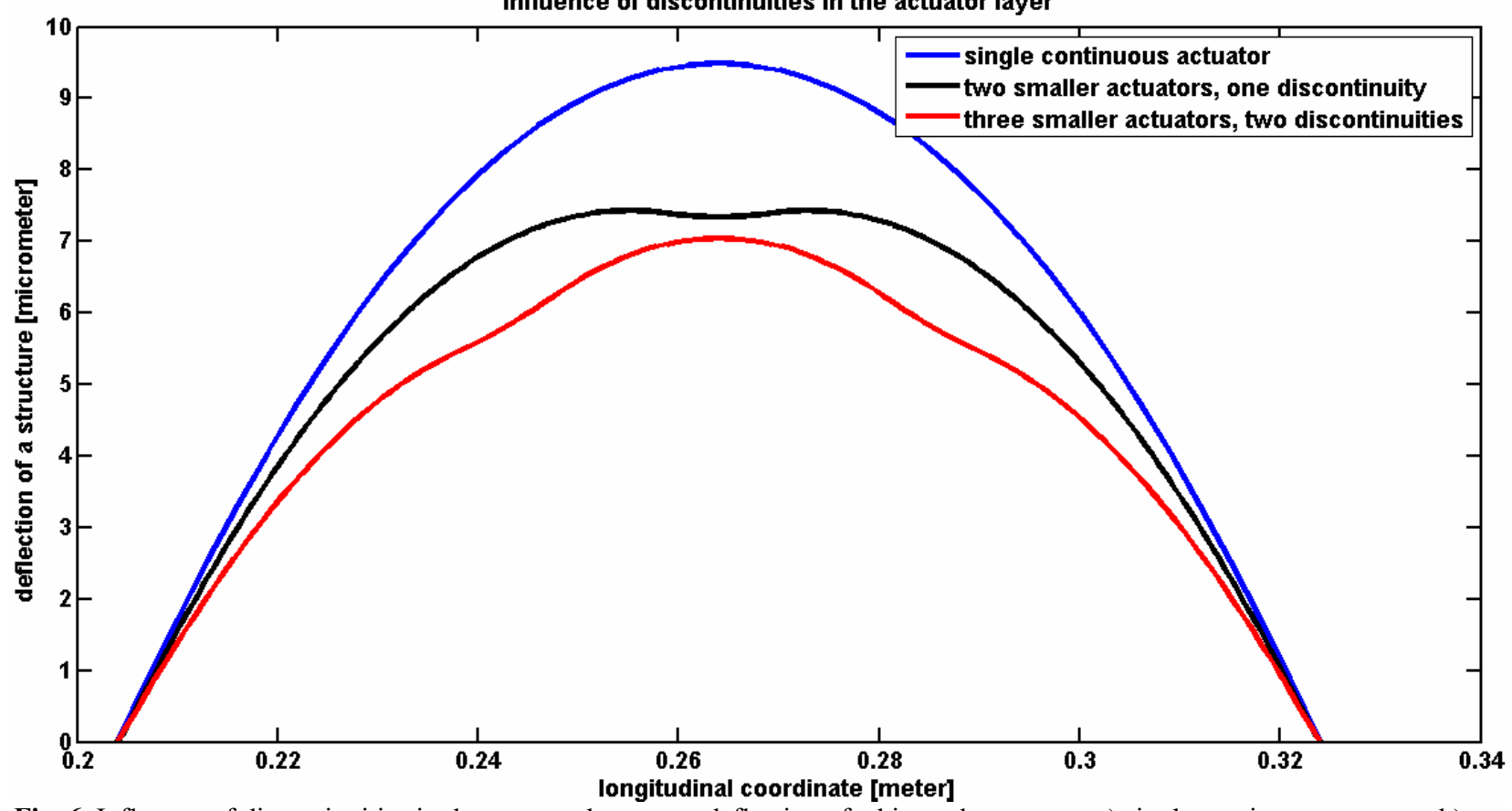

Fig. 6. Influence of discontinuities in the actuator layer on a deflection of a bimorph structure. a) single continuous actuator. b) two smaller actuators with one discontinuity, c) three smaller actuators with two discontinuities.

bending revealed important deformation at discontinuities. Wavefront measurement of a reflected beam shows significant deviation from expected spherical surface at connection point of separate actuators. Measured wavefront and difference from a correct surface is shown at Fig.5. In spite that the deformation is not observable without applied voltage, at $75 \mathrm{~V}$ error it is clearly visible at OPD map and also in calculated far field pattern. When focus is removed, measured error amounts to about $25 \%$ of a full wavefront change.

In order to understand influence of discontinuities in actuator layer, numerical model and test measurement was prepared. Test samples of bending structure were simulated and measurement is in preparation. Samples of 20x120 mm elements were fabricated with single piece, 2 piece and 3 piece actuator layer. Results of calculation are shown in Fig 6. Calculation clearly illustrates grave effect of discontinuities in actuator layer.

\section{Conclusion}

Large-aperture elements for laser active and adaptive optics are investigated in this project. A prototype of lightweight deformable mirror for high-power lasers is currently in development. We are designing, modelling and testing novel scalable size DM based on bimorph architecture with a special composite core and an innovative actuation structure. In order to realize a sufficiently large working aperture, precision and dynamic range we are using new technologies for production of core, bimorph actuator and DM reflector. Detailed simulation of components and structure is validated by measurement and testing. A research of DM actuation and response of a complicated mirror structure needed for an accurate control of a deformation is performed. Testing of samples and subscale measurements are currently performed, measurement of a complete structure is in preparation

\section{Acknowledgement.}

This work was performed with support of Technological grant agency of CR TA01010878.

\section{References}

1 J. T. Salmon, E. S. Bliss, J. L. Byrd, M. Feldman, M. A. Kartz, J. S. Toeppen, B. Vanwonterghem, and S. E. Winter, UCRL-JC-vO33, „First Annual International Conference on Solid-state Lasers for Application to Inertial Confinement Vision ",1995, UCRL-JC-vO33 2 R. A. Zacharias ; N. R. Beer ; E. S. Bliss ; S. C. Burkhart; S. J. Cohen ; S. B. Sutton; R. L. Van Atta ; S. E. Winters ; J. T. Salmon ; M. R. Latta ; C. J. Stolz ; D. C. Pigg ; T. J. Arnold, Opt. Eng. 43(12), pp.2873-2884 (Dec 01, 2004). doi:10.1117/1.1815331

3 J. Ebrardt, JM. Chaput, J.of Physics: Conf.Ser. 112 (2008) 032005 doi:10.1088/1742-6596/112/3/032005 4 Liao, Z. in "The Advanced Maui Optical and Space Surveillance Technologies” 2006, Ed.:S. Ryan, p.E32.

5 S Bonora, C J Hooker, S J Hawkes, J L Collier, C Spindloe, in "Proceedings of the Sixth International Workshop on Adaptive Optics for Industry and Medecine “, Ed. by: Ch. Dainty, Imperial Coledge, ISBN: 978-1-84816-110-8 (hardcover), ISBN: 978-1-84816111-5 (ebook) pp106-111

6 Bruno Le Garrec, in "Light At Extreme IntensitiesOpportunities And Technological Issues Of The Extreme Light Infrastructure“, Lei 2009, AIP Conf. Proc. 1228, pp. 111-116; doi:http://dx.doi.org/10.1063/1.3426039 